\title{
Indeterminacy and the Stability Puzzle in Non-Convex Economies
}

\author{
George W. Evans* \\ University of Oregon
}

\author{
Bruce McGough \\ Oregon State University
}

July 25, 2002

\begin{abstract}
We extend common factor analysis to a multi-dimensional setting by considering a bivariate reduced form model consistent with many Real Business Cycle type models. We show how to obtain new representations of sunspots and find that there are parameter regions in which these sunspots are stable under learning. However, once the parameters are restricted to coincide with those generated by certain standard models of indeterminacy, we find, under one information assumption, that no stable sunspots exist, and under another information assumption, that they exist only for a very small part of the indeterminacy region. This leads to the following puzzle: why does indeterminacy almost always imply instability in RBC-type models?
\end{abstract}

JEL classification: E32, D83, D84.

Keywords: business cycles, sunspots, expectations, learning, stability.

\section{Introduction}

The possibility that business cycle fluctuations are at least in part due to self-fulfilling shifts in private sector expectations was demonstrated in simple dynamic models by (Shell 1977), (Azariadis 1981), (Cass and Shell 1983)

*Financial support form the National Science Foundation is gratefully acknowledged. 
and (Guesnerie 1986). This line of thought has more recently received renewed emphasis in applied stochastic growth models with nonconvexities. In particular, the possibility of multiple equilibria in RBC-type models due to increasing returns, externalities or tax distortions has been emphasized in (Benhabib and Farmer 1994), (Farmer and Guo 1994) and (Farmer 1999). For appropriate parameter regions, such models can have an indeterminate steady state near which there exist "sunspot solutions," i.e. solutions depending on an extraneous exogenous stochastic process. Such a sunspot variable in effect acts as a coordinating device that generates changes in expectations that are self-fulfilling and fully consistent with rational expectations.

A question raised early in the theoretical literature concerns the stability under learning of sunspot equilibria. Are sunspot equilibria plausible in the sense that they could be attained by private agents as a result of an adaptive learning process, such as the least squares updating rules described by (Bray and Savin 1986) and (Marcet and Sargent 1989)? Once again, this possibility was demonstrated first in the context of simple dynamic models: for the overlapping generations model with money, (Woodford 1990) showed that under an adaptive learning rule, the economy could converge to a sunspot equilibrium driven by a two-state Markov process.

Despite extensive theoretical work on the new generation of growth models with nonconvexities, as well as considerable efforts to calibrate such models to the data, relatively little work has been done on the stability under learning of the sunspot solutions to these models. (Evans and Honkapohja 2001), Section 10.5, looked at the standard representation of the sunspot solution in the Farmer-Guo model and found that at the calibrated parameter values this solution was not stable under learning. This finding was confirmed and extended in (Packalén 1999). However, these investigations did not examine the stability of some types of sunspot solutions, as discussed below, nor did they consider recently developed models that are considered more realistic from the viewpoint of empirical calibration.

The current paper undertakes such an investigation and in doing so we focus on two key points. First, we extend the analysis to include new representations of sunspot solutions. A major contribution of this paper is to demonstrate existence and analyze stability of a "common factor" or "resonance frequency" class of stationary sunspot solutions in multivariate models with predetermined variables. This analysis extends some earlier results that found such solutions to be stable under learning in cases where the "general form" sunspot solutions, which are often the focus, are not stable. 
Secondly, in addition to the Farmer-Guo model, which we analyze as a benchmark, we examine stability of sunspot equilibria in two more recent formulations of growth models with nonconvexities, (Benhabib and Farmer 1996) and (Schmitt-Grohé and Uribe 1997). In the two-sector model of (Benhabib and Farmer 1996) it is shown that indeterminacy can arise with much lower degrees of increasing returns than in the Farmer-Guo setup, while (Schmitt-Grohé and Uribe 1997) demonstrate that tax distortions at empirically realistic income tax rates can also generate indeterminacies. ${ }^{1}$

The central results of our analysis are easily summarized. For a general reduced form that includes all three models as special cases, we are able to find large regions of the reduced form parameter space in which there are sunspot solutions that are stable under learning. In some parameter regions these take the form of the general form sunspot solutions that were previously studied, while in other regions it is instead the new common factor solutions that are stable. However, surprisingly, we do not find regions of stability for sunspot solutions in the structural stochastic growth models that we analyze. We call this result the "stability puzzle," because there is no obvious reason for this finding, and the puzzle is intensified by previous results in which we found regions of stability for common factor sunspot solutions in a linearquadratic model of investment with tax or externality distortions.

Our results for the structural models are not entirely negative. The findings just summarized are based on an information assumption, which we find natural, that when forming expectations private agents have available data on all past variables and on current exogenous variables, but not on the current endogenous variables that those expectations in part determine. Stability under learning can, in principle, be affected by allowing expectations to be made contingent on current endogenous variables, and thus, in Section 5 we examine this case as well.

\footnotetext{
${ }^{1}$ After completing a draft of this paper we became aware of the closely related work by (Rudanko 2002). Both papers, which were developed independently, consider stability under learning of sunspot equilibria in irregular RBC-type models, and the main differences are as follows. (Rudanko 2002) focuses on what we call general form sunspot solutions and on minimal state variable solutions, and examines in detail the (Schmitt-Grohé and Uribe 1997) model, including alternative specifications of the fiscal rule. She also investigates stability within the determinacy region. Our paper develops Common Factor representations of sunspot solutions and examines stability for these new solutions as well as for general form sunspots. We investigate an encompassing reduced form model and use it to study the stability of sunspot solutions, within the regions of indeterminacy, for three applied nonconvex RBC models.
} 
For almost all of the parameter space examined the stability results are unchanged. However, now, for each of the models, there is indeed a small part of the indeterminacy region in which common factor sunspot solutions are stable under learning. Clearly this provides hope for the possibility of developing versions of nonconvex growth models, which exhibit sunspot solutions that are robustly stable under learning. However, the stability puzzle

remains: Why is the stability area such a small part of the indeterminacy region, and why is stability in this area sensitive to the detailed information assumptions?

\section{Model and Theory}

We consider the following reduced form model:

$$
\begin{aligned}
c_{t}+e_{1} k_{t} & =f_{2} E_{t}^{*} c_{t+1}+f_{1} E_{t}^{*} k_{t+1}+g_{1} v_{t} \\
k_{t} & =d_{2} c_{t-1}+d_{1} k_{t-1}-g_{2} v_{t-1} \\
v_{t} & =\rho v_{t-1}+u_{t},
\end{aligned}
$$

where $|\rho|<1$ and $u_{t}$ is iid with mean zero and finite variance. In all of the structural growth models we consider below, $k_{t}$ is the logarithm of the capital stock, $c_{t}$ is the logarithm of consumption and $v_{t}$ can be interpreted as a productivity shock. $E_{t}^{*} c_{t+1}$ and $E_{t}^{*} k_{t+1}$ denote forecasts by private agents of $c_{t+1}$ and $k_{t+1}$, respectively, where the expectations are formed in period $t$. These reduced form equations are assumed to determine $c_{t}$ and $k_{t}$ whether or not expectations are formed rationally. Under the standard rational expectations assumption we denote these expectations by $E_{t} c_{t+1}$ and $E_{t} k_{t+1}$, where these are now interpreted as mathematical expectations, conditional on all variables dated $t$ or earlier and given the actual stochastic process followed by $\left(c_{t}, k_{t}, v_{t}\right)$.

Under rational expectations we have $k_{t+1}=E_{t} k_{t+1}$ and we can write

$$
\begin{aligned}
{\left[\begin{array}{ll}
1 & e_{1} \\
d_{2} & d_{1}
\end{array}\right]\left[\begin{array}{l}
c_{t} \\
k_{t}
\end{array}\right] } & =\left[\begin{array}{cc}
f_{2} & f_{1} \\
0 & 1
\end{array}\right]\left[\begin{array}{l}
E_{t} c_{t+1} \\
E_{t} k_{t+1}
\end{array}\right]+\left[\begin{array}{l}
g_{1} \\
g_{2}
\end{array}\right] v_{t} \\
& \text { or } \\
H y_{t} & =F E_{t} y_{t+1}+G v_{t}
\end{aligned}
$$

where $y_{t}=\left(c_{t}, k_{t}\right)^{\prime}$. We begin with an analysis of the solutions under rational expectations (RE). 


\section{$2.1 \quad$ Representations}

In (Evans and McGough 2002) we note the importance of distinguishing between rational expectations equilibria (REE) and their representations (REER); we recall this distinction here. An REE is any stochastic process satisfying the expectational difference equation (3). An REER is a linear difference equation, any solution to which is an REE. In the literature it is standard to identify an REE with its REER; however, as we showed in (Evans and McGough 2002), and as will be the case here, a given REE may have several different REERs. Furthermore, the notion of stability under learning applies to the representations, not the equilibria themselves; and, in some cases, a given equilibrium will have both stable and unstable representations.

For simplicity we take all processes to be doubly infinite, and we restrict attention to stationary REE. ${ }^{2}$ To obtain the standard representation of REE in this model, note that rational expectations implies that $\varepsilon_{t+1}=c_{t+1}-E_{t} c_{t+1}$ satisfies $E_{t} \varepsilon_{t+1}=0$, i.e. $\varepsilon_{t+1}$ is a martingale difference sequence (mds). We can therefore write

$$
y_{t}=F^{-1} H y_{t-1}-F^{-1} G v_{t-1}+\left[\begin{array}{c}
\varepsilon_{t} \\
0
\end{array}\right] \text {. }
$$

This is an REER, which we call the general form representation (or GF solution), and all REE can be represented by (4). ${ }^{3}$

If the eigenvalues of $F^{-1} H$ are inside the unit circle then the general form representation yields stationary solutions for any mds $\varepsilon_{t}$. Thus there are an infinity of equilibria and the model is said to be indeterminate. If precisely one of the eigenvalues is inside the unit circle then there is a unique stationary solution and the model is said to be determinate. If both eigenvalues are outside the unit circle, all solutions are explosive.

In case the matrix $F^{-1} H$ has real eigenvalues with norm less than one the rational expectations equilibria of model (3) have an alternate representation, which we will call a "common factor" representation. This type of representation is nicely motivated by considering the unique REE in the determinate case in which the two eigenvalues satisfy $0 \leq\left|\lambda_{2}\right|<1<\left|\lambda_{1}\right|{ }^{4}$ We require some notation: If $A$ and $B$ are real numbers then their direct sum is

\footnotetext{
${ }^{2}$ All arguments are easily modified to account for initial conditions.

${ }^{3}$ The definition of a general form representation will be modified slightly in Section 2.2.1.

${ }^{4}$ Without loss of generality we are assuming that $\left|\lambda_{1}\right| \geq\left|\lambda_{2}\right|$.
} 
written

$$
A \oplus B=\left[\begin{array}{cc}
A & 0 \\
0 & B
\end{array}\right]
$$

We can stack the model to include the intrinsic shock $v_{t}$ in the state variable to get

$$
\begin{gathered}
{\left[\begin{array}{ccc}
1 & e_{1} & -g_{1} \\
d_{2} & d_{1} & -g_{2} \\
0 & 0 & \rho
\end{array}\right]\left[\begin{array}{l}
c_{t} \\
k_{t} \\
v_{t}
\end{array}\right]=\left[\begin{array}{ccc}
f_{2} & f_{1} & 0 \\
0 & 1 & 0 \\
0 & 0 & 1
\end{array}\right]\left(\left[\begin{array}{c}
c_{t+1} \\
k_{t+1} \\
v_{t+1}
\end{array}\right]+\left[\begin{array}{c}
\varepsilon_{t+1} \\
0 \\
-u_{t+1}
\end{array}\right]\right),} \\
\hat{H} x_{t}=\hat{F}\left(x_{t+1}+\hat{\phi}_{t+1}\right),
\end{gathered}
$$

where the second equation is written to define notation. Now write

$$
\hat{F}^{-1} \hat{H}=S\left(\lambda_{1} \oplus \lambda_{2} \oplus \rho\right) S^{-1}
$$

and notice that $\lambda_{i}$ is an eigenvalue of $F^{-1} H$. Changing coordinates to $z_{t}=$ $S^{-1} x_{t}$, and writing $\eta_{t}=-S^{-1} \hat{\phi}_{t}$, the solution becomes

$$
z_{t}=\left(\lambda_{1} \oplus \lambda_{2} \oplus \rho\right) z_{t-1}+\eta_{t}
$$

In the determinate case $\left|\lambda_{1}\right|>1$. For the process (5) to be stationary, $z_{1 t}$ must then be zero; accordingly, the martingale difference sequence $\varepsilon_{t}$ must be such that $\eta_{1 t}=0$. Imposing this requirement restricts the dynamics of the solution process to the contracting eigenspace, and the corresponding process is stationary. ${ }^{5}$ This method yields the unique (stationary) REE and selects the same solution as the method developed by (Blanchard and Kahn 1980). Note that in this case the solution does not depend on sunspots, i.e. on exogenous variables other than $v_{t}{ }^{6}$

One can proceed from (5) slightly differently, and obtain a method of solution that generalizes to the indeterminate case. Rewrite (5) as

$$
\left(1-\lambda_{1} L\right) \oplus\left(1-\lambda_{2} L\right) \oplus(1-\rho L) z_{t}=\eta_{t}
$$

\footnotetext{
${ }^{5}$ In case of models with initial conditions, the initial value of the free variables must be chosen so that the corresponding state vector lies in the contracting eigenspace.

${ }^{6}$ To see this note that $z_{1 t} \equiv 0$ implies that $c_{t}$ is a linear function of $k_{t}$ and $v_{t}$. This relationship, together with the second two equations from (1), fully specify the solution.
} 
Restricting $\varepsilon_{t}$ as above, we get that $\eta_{1 t}=0$. Now act on each side of the equation by $\left(1-\lambda_{1} L\right)^{-1} \oplus 1 \oplus 1$ so that equation (6) becomes $^{7}$

$$
1 \oplus\left(1-\lambda_{2} L\right) \oplus(1-\rho L) z_{t}=\eta_{t}
$$

Notice that since $\eta_{1 t}=0$, by dividing out $\left(1-\lambda_{1} L\right)$, we deny the solution $z_{1 t}=\lambda_{1} z_{1 t-1}$. Thus we have restricted the dynamics just as above and the corresponding solution is the unique REE in the determinate case.

We call the method of solution described in the preceding paragraph "common factor analysis". This method does not require a determinate model, and, in fact, becomes more interesting in the indeterminate case, as we proceed to discuss now. Assume both eigenvalues are real and are inside the unit circle. Equation (5) still holds and the solution is stationary for any $\varepsilon_{t}$. We can again act on each side of the equation by $\left(1-\lambda_{1} L\right)^{-1} \oplus 1 \oplus 1$, but this time we can permit $\eta_{1 t} \neq 0$, so that the solution $z_{1 t}=\lambda_{1} z_{1 t-1}$ is not discarded; rather, the corresponding dynamics are captured by

$$
z_{1 t}=\left(1-\lambda_{1} L\right)^{-1} \eta_{1 t}=\xi_{t},
$$

where the second equality defines notation. We can now untangle the coordinates to discover the implications of this equation for our original state variables. Not surprisingly, we have the exogenous noise term and the predetermined capital motion expressed as before. However, using the definition of $z_{1 t}$, now we know to write consumption as

$$
c_{t}=-\frac{S^{12}}{S^{11}} d_{2} c_{t-1}-\frac{S^{12}}{S^{11}} d_{1} k_{t-1}+\frac{S^{12}}{S^{11}} g_{2} v_{t-1}-\frac{S^{13}}{S^{11}} v_{t}+\xi_{t},
$$

where $S^{i j}$ is the $i j$-component of $S^{-1}$. We summarize these results in the following proposition, using the fact that $S$ can be normalized so that $S^{i 1}=1$ for $i=1,2$.

Proposition 1 Assume the eigenvalues $\lambda_{i}$ of $F^{-1} H$ are real with norm less than one. Let $\varepsilon_{t}$ be any martingale difference sequence, $\eta_{1 t}=\varepsilon_{t}+S^{i 3} u_{t}$, and

$$
\xi_{t}=\lambda_{i} \xi_{t-1}+\eta_{1 t}
$$

\footnotetext{
${ }^{7}$ Technically, $\left(1-\lambda_{1} L\right)$ is not invertible; but the argument can be made formally by using the fact that $\eta_{1 t}=0$ to rewrite equation (6) with $\left(1-\lambda_{1} L\right) \oplus 1 \oplus 1$ modifying $\eta_{t}$. Then it is clear that (7) implies (6) so that (7) defines an REE.
} 
Then

$$
y_{t}=\left[\begin{array}{cc}
-S^{i 2} d_{2} & -S^{i 2} d_{1} \\
d_{2} & d_{1}
\end{array}\right] y_{t-1}+\left[\begin{array}{c}
\xi_{t} \\
0
\end{array}\right]+\left[\begin{array}{c}
S^{i 2} g_{2} \\
g_{2}
\end{array}\right] v_{t-1}-\left[\begin{array}{c}
S^{i 3} \\
0
\end{array}\right] v_{t}
$$

is an REER and all REE can be represented by (9).

We call such REERs Common Factor Representations (or CF solutions). If $\varepsilon_{t}=-S^{i 3} u_{t}$ then $\xi_{t}=0$ and the resulting REER is often called a minimum state variable solution. ${ }^{8}$ Notice that in the determinate case $\left|\lambda_{1}\right|>1$, so that $\varepsilon_{t}$ must be chosen equal to $-S^{13} u_{t}$ and the above REER then determines the unique REE. In contrast, in the indeterminate case $\varepsilon_{t}$ is not restricted, and the minimal state variable solutions are special cases of the common factor solutions.

\subsection{T-maps}

A useful way to analyze representations of REE's is to view them as fixed points of a map. Specifically, assume that agents know the functional form of the representation, but do not know the corresponding parameter values; we assume they have some perception of what the parameter values are and we call the subsequent functional form with these values imposed the perceived law of motion (PLM). Agents use the PLM to form their expectations of future values of $y_{t}$. The actual law of motion (ALM) is obtained by inserting these expectations into the reduced form model. Provided the PLM is wellspecified, the ALM will have the same functional form and the associated parameter values, which we think of as the actual parameter values, will depend on the agents' perceptions. If $\theta$ represents the agents' perceived parameter values, let $T(\theta)$ represent the actual parameter values. Then a fixed point of this $T-$ map determines an REER.

This mapping, from PLM to ALM parameters, plays a central role in the analysis of stability under learning. Under least squares learning the PLM parameters are assumed to be estimated by least squares regressions and the parameter estimates updated over time as new data are generated. The question of interest is whether these parameters can converge to an REER

\footnotetext{
${ }^{8}$ For further discussion of minimal state variable solutions see (McCallum 1983) and chapters 8-10 of (Evans and Honkapohja 2001). McCallum also advocates a subsidiary selection criterion, whereas we instead examine stability under learning.
} 
fixed point, and as is discussed below, this question can be answered by examining the properties of the T-map.

Because of the different structure of general form and common factor representations, we will assume PLMs that have functional forms tailored specifically for each type. It is possible to write down a PLM so that the corresponding T-map has, as fixed points, both general form and common factor solutions; however, stability under learning of a particular type of representation would subsequently require a stronger form of stability than is typically sought; specifically, the stability of the REER would have to be robust to over-parameterization. Since the principal stability results of this paper are negative, it makes sense to focus on the weaker notion of stability, and thus we ignore such robustness issues here.

There is one further subtlety that arises in connection with obtaining the map from PLM to ALM parameters, which is that the T-map will be affected by the detailed information set available to agents when forecasts are made. Of course, REE themselves can be affected by the information assumed available to agents, but the issue is even more acute when studying out of equilibrium learning behavior. The main issue is whether expectations can be conditioned on current endogenous variables, as well as on current exogenous variables; although under RE these are equivalent assumptions, they are not equivalent outside of RE. Throughout the remainder of this Section, and in Sections 3 and 4, we assume that agents do not condition on current values of the endogenous variables. In Section 5 we take up the alternative information assumption.

\subsubsection{General Form PLMs}

To capture general form representations we postulate for our agents the following PLM:

$$
c_{t}=a c_{t-1}+b k_{t-1}+c+d v_{t-1}+e v_{t}+f \varepsilon_{t} .
$$

Here $\varepsilon_{t}$ is the observable sunspot. We take the functional form of the capital accumulation equation as known. Thus agents are aware that

$$
k_{t}=d_{1} k_{t-1}+d_{2} c_{t-1}-g_{2} v_{t-1} \text {. }
$$

As indicated above and in the Introduction, the precise information assumptions can affect learning stability. Our first information assumption will be 
that when they form their expectations private agents have available data on all past variables and on current exogenous variables, $v_{t}$ and $\varepsilon_{t}$, but not on the current endogenous variables $c_{t}$ and $k_{t}$ that these expectations in part determine. Using their PLM, expectations are thus formed as

$$
\begin{aligned}
& E_{t}^{*} c_{t+1}=a E_{t}^{*} c_{t}+b E_{t}^{*} k_{t}+c+d v_{t} \\
& E_{t}^{*} k_{t+1}=d_{1} E_{t}^{*} k_{t}+d_{2} E_{t}^{*} c_{t}-g_{2} v_{t}
\end{aligned}
$$

where

$$
\begin{aligned}
& E_{t}^{*} c_{t}=a c_{t-1}+b k_{t-1}+c+d v_{t-1}+e v_{t}+f \varepsilon_{t} \\
& E_{t}^{*} k_{t}=d_{1} k_{t-1}+d_{2} c_{t-1}-g_{2} v_{t-1} .
\end{aligned}
$$

Thus $E_{t}^{*} c_{t+1}$ and $E_{t}^{*} k_{t+1}$ are linear functions of $\left(c_{t-1}, k_{t-1}, 1, v_{t}, v_{t-1}, \varepsilon_{t}\right)$.

To obtain the T-map from PLM to ALM parameters we insert these expectations into (1) to obtain the implied equation for $c_{t}$ as a linear function of $\left(c_{t-1}, k_{t-1}, 1, v_{t-1}, v_{t}, \varepsilon_{t}\right)$. The associated T-map is computed to be

$$
\begin{aligned}
a & \rightarrow-e_{1} d_{2}+f_{1}\left(d_{1} d_{2}+d_{2} a\right)+f_{2}\left(a^{2}+b d_{2}\right) \\
b & \rightarrow-e_{1} d_{2}+f_{1}\left(d_{1}^{2}+d_{2} b\right)+f_{2}\left(a b+b d_{1}\right) \\
c & \rightarrow c\left(f_{1} d_{2}+f_{2}(1+a)\right) \\
d & \rightarrow e_{1} g_{2}+f_{1}\left(d_{2} d-d_{1} g_{2}\right)+f_{2}\left(a d-b g_{2}\right) \\
e & \rightarrow g_{1}+f_{1}\left(d_{2} e-g_{2}\right)+f_{2}((a+\rho) e+d) \\
f & \rightarrow\left(f_{2} a+f_{1} d_{2}\right) f .
\end{aligned}
$$

Let $\theta=[a, b, c, d, e, f]^{\prime}$. Any solution to the system of equations $T(\theta)=\theta$ defines an REER. The subsystem (12) and (13) decouples from the rest and so can be solved separately. This subsystem simplifies into a cubic in $a$ and the corresponding solutions are given as

$$
a_{0}=\frac{1-d_{2} f_{1}}{f_{2}}, b_{0}=\frac{e_{1}-d_{1} f_{1}}{f_{2}} \quad \text { and } \quad a_{i}=S^{i 2} d_{2}, b_{i}=S^{i 2} d_{1}, i=1,2 .
$$

We proceed to analyze these solutions separately.

Case 1: $a=a_{0}, b=b_{0}$. In this case, $c=0$ and $f_{2} a+f_{1} d_{1}=1$. This implies that $d$ and $f$ are free and

$$
e=\frac{f_{2} d-f_{1} g_{2}+g_{1}}{1-f_{2}(a+\rho)-f_{1} d_{2}}
$$


Let $\Omega_{G F}$ be the set of all fixed points of $T$ so that $\left(\theta_{1}, \theta_{2}\right)=\left(a_{0}, b_{0}\right)$, and notice that $\Omega_{G F}$ is a two dimensional affine subspace. We would like to identify general form representations with elements of $\Omega_{G F}$; however, there is one caveat, which requires us to slightly modify our definition. Because $d$ is free, it will not necessarily coincide with the coefficient of $v_{t-1}$ as determined by (4). ${ }^{9}$ To avoid this complication, we generalize our definition slightly and say that for fixed mds $\varepsilon_{t}$, an REER is a general form representation if its coefficients can be identified with a fixed point in $\Omega_{G F}$. Notice this includes all representations of the form (4).

Case 2: $a=a_{i}, b=b_{i}$. Again we have that $c=0$, but in this case, $f_{2} a+f_{1} d_{1} \neq 1$ so that $f=0$ as well. Also, $d$ and $e$ are pinned down and algebra shows these fixed points correspond to the minimum state variable solutions.

\subsubsection{Common Factor PLMs}

Common factor representations require a PLM of the following form:

$$
c_{t}=a c_{t-1}+b k_{t-1}+c+d v_{t-1}+e v_{t}+f \xi_{t},
$$

where it is assumed that $\xi_{t}$ is observable and follows a process of the form

$$
\xi_{t}=\lambda_{i} \xi_{t-1}+\eta_{1 t}
$$

Expectations of $c$ are now formed as

$$
\begin{aligned}
E_{t}^{*} c_{t+1} & =a E_{t}^{*} c_{t}+b E_{t}^{*} k_{t}+c+d v_{t}+f \lambda_{i} \xi_{t} \\
E_{t}^{*} c_{t} & =a c_{t-1}+b k_{t-1}+c+d v_{t-1}+e v_{t}+f \xi_{t},
\end{aligned}
$$

where $E_{t}^{*} k_{t}$ and $E_{t}^{*} k_{t+1}$ are as before.

The corresponding $T-$ map is given by items (12)-(16) above and

$$
f \rightarrow\left(f_{1} d_{2}+f_{2}\left(a+\lambda_{i}\right)\right) f .
$$

\footnotetext{
${ }^{9}$ Of course there is an alternative mds, $\varepsilon_{t}^{\prime}$, so that the corresponding REER satisfies (4) exactly. If $d=\left(f_{1} g_{2}-g_{1}\right) / f_{2}$ then, by (18), $e=0$ and the result follows for the original mds. If $d$ is some other real number then $v_{t}=\rho v_{t-1}+u_{t}$ implies

$$
d v_{t-1}+e v_{t}=(d+e \rho) v_{t-1}+e u_{t},
$$

which shows this fixed point defines a general form REER with sunspot mds $\varepsilon_{t}^{\prime}=f \varepsilon_{t}+e u_{t}$.
} 
Case 1: $a=a_{0}, b=b_{0}$. Here $f=0, d$ is free, and $e$ is pinned down by the choice of $d$. This fixed point corresponds to REER which capture general form solutions that depend only on intrinsic noise, though, in case $e \neq 0$, these solutions are not minimal.

Case 2: $a=a_{i}, b=b_{i}$. Now $d$ and $e$ are determined and $a_{i}+\lambda_{i}=a_{0}$. This implies $f$ is free and algebra shows these fixed points correspond to the common factor representations. Note that if $f=0$ then the fixed point corresponds to a minimum state variable solution.

Let $\Omega_{C F, i}$ be the set of fixed points of the T-map so that $\left(\theta_{1}, \theta_{2}\right)=\left(a_{i}, b_{i}\right)$, and notice that $\Omega_{C F, i}$ is a one dimensional affine subspace. We identify $i$-th root common factor representations with points in $\Omega_{C F, i}$.

\section{$2.3 \quad$ E-stability}

Let $\theta^{*}$ be a fixed point of the T-map. We say $\theta^{*}$ (and the associated REER) is $E$-stable provided the differential equation

$$
\frac{d \theta}{d \tau}=T(\theta)-\theta
$$

is locally asymptotically stable at $\theta^{*}$. The E-stability Principle says that if the REER is E-stable then it is learnable by a reasonable algorithm such as recursive least squares. ${ }^{10}$ The definition of expectational stability just given is inadequate when there is a non-trivial connected set of rest points of the differential equation (23), as is the case for our model; if $\Omega$ is locally connected then no point in $\Omega$ is locally asymptotically stable. In this case the notion of E-stability is extended as follows: we say that a set of fixed points, $Q$, is E-stable provided there is a neighborhood $U$ of $Q$ so that for any $\theta_{0} \in U$ the trajectory of $\theta$ determined by the differential system (23) converges to a point in $Q$. A necessary condition for E-stability of $Q$ is that for all $q \in Q$ the non-zero eigenvalues of the derivative $T(\theta)-\theta$ evaluated at $q$ have negative real part. Sufficient conditions are more difficult to obtain because of the presence of zero eigenvalues.

\footnotetext{
${ }^{10}$ For a general discussion of the connection between E-stability and least-squares learning see (Evans and Honkapohja 2001). (Evans and McGough 2002) provides numerical support, for the types of solution discussed here, within a univariate set-up.
} 


\subsubsection{E-stability of General Form PLMs}

The decoupled nature of the T-map allows us to analyze separately the stability of certain subsystems. The relevant derivatives are given below:

$$
\begin{aligned}
D T_{a b} & =\left[\begin{array}{cc}
2 f_{2} a+f_{1} d_{2} & f_{2} d_{2} \\
f_{2} b & f_{2}\left(a+d_{1}\right)+f_{1} d_{2}
\end{array}\right] \\
D T_{c} & =f_{2}(1+a)+f_{1} d_{2} \\
D T_{d} & =f_{2} a+f_{1} d_{2} \\
D T_{e f} & =\left[\begin{array}{cc}
f_{2}(a+\rho)+f_{1} d_{2} & 0 \\
0 & f_{1} d_{2}+f_{2} a
\end{array}\right]
\end{aligned}
$$

Notice that these derivatives are invariant over the set $\Omega_{G F}$. From the definition above, the set $\Omega_{G F}$ satisfies the necessary condition for E-stability provided the eigenvalues of these derivatives have real part less than or equal to one. In this case, slightly abusing terminology, we will call the set $\Omega_{G F}$ E-stable. If $\Omega_{G F}$ is E-stable, we also say that the general form representation is stable under learning.

\subsubsection{E-stability of Common Factor PLMs}

Analysis similar to the above can be preformed in case of common factor PLMs. The only difference is $D T_{e f}$ and the new form of this derivative is given here.

$$
D T_{e f}=\left[\begin{array}{cc}
f_{2}(a+\rho)+f_{1} d_{2} & 0 \\
0 & f_{1} d_{2}+f_{2}\left(a+\lambda_{i}\right)
\end{array}\right]
$$

As above, these derivatives are invariant over the set $\Omega_{C F, i}$. The one dimensional nature of the set $\Omega_{C F, i}$ together with the form of the system of differential equations implies that a necessary and sufficient condition for $\Omega_{C F, i}$ to be E-stable is that all eigenvalues of the derivative have real part less than or equal to one and only one eigenvalue has real part equal to one; for details on this result, see p. 6 of (Evans and Honkapohja 1992). If $\Omega_{C F, i}$ is E-stable, we say that the $i$-th root common factor representation is stable under learning.

We remark that for both PLMs our definition of E-stability, and the Estability conditions given, include convergence of the intercept term. While its REE value is equal to zero in the linearization given, this is simply because 
variables have been expressed as (log) deviations from their steady state values. Since the intercept value in the PLM, for variables not expressed in deviation form, depends on these steady state values as well as on structural parameters, it is most natural to assume that the intercept as well as slope parameters would need to be estimated under least squares learning. As noted in the earlier literature, including stability of the intercept coefficient can in some cases be a binding requirement.

\section{$3 \quad$ Stability in the Reduced Form Model}

The first piece of the stability puzzle comes from the analysis of the reduced form model itself. We ask whether there are parameter values for which the model is indeterminate and for which either the general form representation or one or both of the common factor representations is stable under learning. We find the answer is a resounding yes; in fact, regions of stability are not difficult to find. Unfortunately, the algebraic complexity of the indeterminacy and stability conditions makes an analytic characterization of parameters for which both stability and indeterminacy hold intractable. Also, because the parameter space is seven dimensional, a complete numerical analysis is not feasible. We are therefore forced to report numerical results for a lattice over a small region in parameter space.

To choose parameters in a somewhat meaningful way, we take as our benchmark a standard calibration of the Benhabib-Farmer model; see Section 4.3 below for details. The corresponding reduced form parameter values are given by

$$
\begin{aligned}
& d_{1}=1.078, d_{2}=-0.2201, e_{1}=-1.0821, f_{1}=-1.0524, \\
& f_{2}=0.8537, g_{1}=0.3839, g_{2}=0.2942 .
\end{aligned}
$$

We note that these reduced form parameters result in unstable sunspot equilibria.

Intuitively, the parameters most likely to affect stability and determinacy are those modifying the expectations terms. Thus, to search for regions of stability, we allow $f_{1}$ and $f_{2}$ to vary between -2 and 2 with step size .04, and hold the remaining parameters fixed. We then check for indeterminacy and in case it holds, we check the stability of $\Omega_{G F}$. Finally, we check for existence of CF-solutions and in case they exist, we analyze the stability of $\Omega_{C F, i}$. We find that for $f_{1} \in[-.4,2]$ there are multiple corresponding values 
of $f_{2}$ for which exactly one of the common factor sunspots is E-stable, and that the corresponding values of $f_{2}$ are always negative and range from -.9 to -1.9 ; for example, $f_{1}=-.4$ and $f_{2}=-.92$ yields an E-stable sunspot equilibrium. Also, we find that for no parameter values is the general form solution stable.

To obtain more general results we allowed all parameters to vary by considering a coarse lattice around the origin; the computational intensity of this exercise prohibits a more careful analysis. Besides regions of instability for all types of representations, we found regions for which each of the following was true:

1. Exactly one of the CF-solutions is stable and the GF-solution is unstable.

2. Both CF-Solutions are stable and the GF-solution is unstable.

3. Both CF-solutions are unstable and the GF-solution is stable.

4. The GF-solution is stable and exactly one of the CF-solutions is stable. ${ }^{11}$

These results suggest that stable sunspots can exist in applied models that yield this reduced form, and that cases of stable sunspot solutions may well take the specific form of stable CF-solutions. The key to obtaining to obtaining stability of CF-solutions, in regions where GF-solutions are not stable, is for agents to use a PLM that conditions on an exogenous $\operatorname{AR}(1)$ process $\xi_{t}$ with precisely the right autoregressive parameter. Such an exogenous process can be described as having a "resonant frequency" since its time series properties are just right for generating stable sunspot fluctuations. The term "resonant frequency condition" was used in (Evans and Honkapohja 2002) and (Evans, Honkapohja, and Marimon 2001), in connection with finite state Markov sunspots, to describe the (well-known) condition required on their transition probabilities. For univariate models, (Evans and McGough 2002) showed that this phenomenon extends to more general exogenous processes and to models with predetermined variables.

\footnotetext{
${ }^{11}$ Recall that we say the GF-solution is stable when the set $\Omega_{G F}$ satisfies the necessary condition for E-stability.
} 


\section{Instability in Applied Models}

The second piece of the stability puzzle comes from the analysis of applied models that yield our reduced form. We study three well known Real Business Cycle type models: the Farmer-Guo Model (FG-model), the SchmittGrohé-Uribe Model (SGU-model), and the Benhabib-Farmer Model (BFmodel). We begin by outlining the theory behind the model, and then derive maps from the models' structural parameters to the reduced form parameters. Then, for different values of the structural parameters, we consider the stability of general form and common factor sunspots in the corresponding reduced form model. We find, in all cases, that all sunspot equilibria are unstable. $^{12}$

\subsection{The Farmer-Guo Model}

We begin with this model because it is a well-known benchmark. To obtain a model exhibiting indeterminacy, Farmer and Guo rely on increasing returns to scale in certain production functions. There are several interpretations of the model; we base ours on monopolistic competition as is consistent with (Farmer and Guo 1994).

\subsubsection{Theory}

Assume the economy is populated by a continuum of identical agents who are consumer-producers. Each agent produces a unique intermediate good. The intermediate goods are then combined using an aggregation technology and sold competitively as a single final good. The utility function of the representative consumer is given by

$$
U=E \sum_{t=1}^{\infty} \beta^{t-1}\left[\log \left(C_{t}\right)-\frac{L_{t}^{1+\chi}}{1+\chi}\right]
$$

and consumer $i$ maximizes this utility subject to the constraint

$$
C_{i t}+K_{i t+1} \leq K_{i t}(1-\delta)+P_{i t} Y_{i t}
$$

\footnotetext{
${ }^{12}$ Recall the timing assumption in this section that expectations are not conditioned on the current value of the endogenous variable.
} 
The final summand on the right hand side of this budget constraint represents the income received by the $i^{t h}$-consumer-producer for producing $Y_{i t}$ units of her intermediate good; $P_{i t}$ is price relative to final goods. Assume the aggregation technology is given by

$$
Y_{t}=\left(\int Y_{i t}^{\lambda} d i\right)^{1 / \lambda}
$$

Perfect competition in the final goods market implies

$$
P_{i t}=\left(\frac{Y_{i t}}{Y_{t}}\right)^{\lambda-1} \text {. }
$$

The firm of the $i^{\text {th }}$-producer is small and so she takes the aggregate output level as given; but she does have market power so that her problem can be written

$$
\max \left\{\left(\frac{Y_{i t}}{Y_{t}}\right)^{\lambda-1} Y_{i t}-\omega_{t} L_{i t}-r_{t} K_{i t}\right\}
$$

where $\omega_{t}$ is the competitive wage and $r_{t}$ is the competitive rental rate on capital. The producer's production function is given by

$$
y_{i t}=V_{t} K_{i t}^{\mu}\left(\gamma^{t} L_{i t}\right)^{\nu}
$$

where $V_{t}$ is a technology shock, $\gamma$ represents growth, and $\mu+\nu>1$ indicating increasing returns to scale. Concavity of the objective function is guaranteed provided $\lambda(\mu+\nu) \leq 1$.

Solving the agent's problem, combining and aggregating yields the following five equations describing the aggregate economy:

$$
\begin{aligned}
K_{t+1} & =Y_{t}+(1-\delta) K_{t}-C_{t} \\
Y_{t} & =V_{t} K_{t}^{\mu}\left(\gamma^{t} L_{t}\right)^{\nu} \\
n \frac{Y_{t}}{L_{t}} & =C_{t} \\
\frac{1}{C_{t}} & =\beta E_{t}\left[\frac{1}{C_{t+1}}\left(1-\delta+m \frac{Y_{t+1}}{K_{t+1}}\right)\right] \\
V_{t} & =V_{t-1}^{\rho} S_{t}
\end{aligned}
$$

where $m=\lambda \mu$ and $n=\lambda \nu$.

To obtain a reduced form model, normalize the variables by dividing out the growth and then log-linearize around the steady state; see the Appendix for details. 


\subsubsection{Results}

Following (Farmer and Guo 1994), we take $\beta=.99, \delta=.025, \chi=0$, and $\gamma=1$. The standard RBC model is obtained with $\lambda=1$ indicating competitive markets, and $\nu=1-\mu$ indicating constant returns to scale. With these parameter values the model is determinate so that sunspots do not exist, and the unique equilibrium is stable under learning. ${ }^{13}$ To obtain indeterminacy in their model, Farmer-Guo choose $\lambda=.58, \mu=.4$, and $\nu=1.21$. These parameters correspond to firms having market power and the technology exhibiting increasing returns to scale. The subsequent model is indeed indeterminate so that sunspots exist. However, these sunspots are not stable under learning. Instability of the general form solution was reported by (Evans and Honkapohja 2001), Section 10.5. Furthermore, for these parameters, the relevant eigenvalues are complex so that common factor solutions do not exist. Setting $\nu=1.1$ yields real eigenvalues and the model remains indeterminate so that common factor sunspots do exist, but, again, they are not stable under learning.

We analyzed a lattice over a region of parameter space corresponding to $\lambda(\mu+\nu)=1$ for $\lambda \in(.2,1]$ and $\mu>0$. These restrictions were chosen to obtain the RBC model as a limiting case.

\section{FIGURE 1 ABOUT HERE}

The results are reported in Figure 1. Here, marked lattice points correspond to indeterminate models. A lattice point marked by an " $\times$ " or "O" indicates existence of common factor representations (the distinction will be discussed in Section 5), and a lattice point marked by a dot indicates only general form solutions exist. Points east of the eastern boundary of the pictured lattice correspond to parameter pairs for which the corresponding value of $\mu$ was negative, and points west of the western boundary correspond to parameter pairs for which the model is determinate. None of the point analyzed yielded a stable representation.

\footnotetext{
${ }^{13}$ In the determinate case, the equilibrium is obtained by requiring agents to choose initial consumption on the stable saddle path. Note that common factor analysis yields this solution by advising the modeler to divide out the explosive eigenspace.
} 


\subsection{The Schmitt-Grohé \& Uribe Model}

To obtain an indeterminate model for calibrated values of the structural parameters, (Schmitt-Grohé and Uribe 1997) consider a simple RBC model with potentially income-elastic government spending and endogenous capital and labor income tax rates. While for calibration purposes they considered four policy methods, for simplicity we consider only the case of fixed exogenous government spending which, in their paper, is denoted Policy 1. We use a discrete time version of their model.

\subsubsection{Theory}

This model is a simple modification of a standard RBC model. Agents have the same utility as specified in the FG-model and maximize this subject to a budget constraint which includes labor income tax rate $\tau_{t}^{l}$ and capital income tax rate $\tau_{t}^{k}$. The latter is levied on capital income after depreciation. Firms have access to a Cobb-Douglas production function $Y=K^{m} L^{1-m}$, and behave competitively so that capital and labor are paid their marginal products. Government spending is assumed fixed and the budget is balanced so that the government's budget constraint is given by

$$
g=\tau_{t}^{l} w_{t} L_{t}+\tau_{t}^{k}\left(r_{t}-\delta\right) K_{t}
$$

To simplify matters, we assume, as did Schmitt-Grohé-Uribe, that the ratio of tax rates is constant: $\tau_{t}^{k}=R \tau_{t}^{l}$. Letting $\tau_{t}=\tau_{t}^{l}$ be the endogenous labor income tax rate and imposing the equilibrium conditions results in the following equations describing the dynamics of the economy:

$$
\begin{aligned}
g & =(1+(R-1) m) \tau_{t} Y_{t}-\delta R \tau_{t} K_{t} \\
K_{t+1} & =Y_{t}+(1-\delta) K_{t}-C_{t}-g \\
Y_{t} & =v_{t} K_{t}^{m} L_{t}^{(1-m)} \\
C_{t} L_{t}^{\chi+1} & =(1-m) Y_{t}\left(1-\tau_{t}\right) \\
C_{t}^{-1} & =\beta E_{t} C_{t+1}^{-1}\left(1+\left(m \frac{Y_{t+1}}{K_{t+1}}-\delta\right)\left(1-R \tau_{t+1}\right)\right)
\end{aligned}
$$

The reduced form model is obtained by log-linearizing about the steady-state; see the Appendix for details. 


\subsubsection{Results}

Following SGU, we take $\beta=.96, \chi=0, m=.3$ and $\delta=.1$ (annual depreciation rate and discount factor. $)^{14}$ Two exogenous parameters remain: fixed government spending $g$; and the fixed tax ratio $R$.

\section{FIGURE 2 ABOUT HERE}

SGU report the numerically computed region of indeterminacy for their model in terms of the endogenously determined tax rates, and subsequently, we do as well; this region, as determined by our computations is reported in Figure 2, and is broadly consistent with the region reported by SGU [See page 987 of (Schmitt-Grohé and Uribe 1997)]. ${ }^{15}$ For convenience, a nonlinear lattice structure was used and the lattice points are marked as in Figure 1. For reference, we include the approximate income tax rates of Canada, US, UK, Japan, Italy, Germany, and France as reported by SGU. Notice that some countries are quite near values for which common factor solutions exist; however, all sunspot representations obtained from the SGU-model are unstable under learning. ${ }^{16}$

\subsection{The Benhabib-Farmer Model}

The Farmer-Guo model has been criticized because the degree of increasing returns required for indeterminacy can not be empirically supported. To address this criticism, (Benhabib and Farmer 1996) develop a model that relies on sector specific externalities to generate regions of indeterminacy. We consider this model here.

\subsubsection{Theory}

Assume the economy is populated by a continuum of agents who are consumerproducers. Each agent owns a firm that produces consumption goods $C$ and

\footnotetext{
${ }^{14}$ To create Figure 2 , we used $\beta=.99$ and $\delta=.025$; these being the quarterly analogues of SGU's annual calibrations. A quarterly calibration of our discrete model yields results that more closely approximate the continuous time model analyzed by SGU.

${ }^{15}$ Some differences can be expected due to our use of a discrete time model.

${ }^{16}$ The values for the UK and Japan were clearly in the indeterminacy region as reported by SGU; however, they appear to lie on the boundary of our region of indeterminacy. Again, we attribute this to our discretation of their model.
} 
investment goods $I$ according to the following constant returns to scale technologies:

$$
\begin{aligned}
C_{i t} & =A_{t} V_{t}\left(K_{i t}^{C}\right)^{m}\left(L_{i t}^{C}\right)^{n} \\
I_{i t} & =B_{t} V_{t}\left(K_{i t}^{I}\right)^{m}\left(L_{i t}^{I}\right)^{n}
\end{aligned}
$$

where $A$ and $B$ are defined by

$$
\begin{aligned}
& A_{t}=\left[\int\left(K_{i t}^{C}\right)^{m}\left(L_{i t}^{C}\right)^{n} d i\right]^{\theta} \\
& B_{t}=\left[\int\left(K_{i t}^{I}\right)^{m}\left(L_{i t}^{I}\right)^{n} d i\right]^{\theta}
\end{aligned}
$$

and represent sector specific externalities, and $V_{t}$ represents technology shocks. Agents are assumed to maximize the same utility function as presented in the Farmer-Guo model. Factor markets are assumed competitive; optimizing behavior by the firm then implies a budget constraint of the form

$$
C_{i t}+\frac{A_{t}}{B_{t}}\left(K_{i t+1}-(1-\delta) K_{i t}\right) \leq A_{t} Y_{i t},
$$

where $Y_{i t}=V_{t} K_{i t}^{m} L_{i t}^{n}$ and $m+n=1$.

The following six equations describe the aggregate behavior of the economy:

$$
\begin{aligned}
K_{t+1} & =(1-\delta) K_{t}+I_{t} \\
Y_{t} & =C_{t}^{\phi}+I_{t}^{\phi} \\
Y_{t} & =V_{t} K_{t}^{m} L_{t}^{n} \\
Y_{t} & =\frac{1}{n} C_{t}^{\phi} L_{t}^{\chi+1} \\
I_{t}^{-\theta \phi} C_{t}^{-\phi} & =\beta E_{t}\left[I_{t+1}^{-\theta \phi} C_{t+1}^{-\phi}\left(1-\delta+m I_{t+1}^{\theta \phi} \frac{Y_{t+1}}{K_{t+1}}\right)\right] \\
V_{t} & =V_{t-1}^{\rho} S_{t}
\end{aligned}
$$

where $\phi=1 /(1+\theta)$. To obtain the reduced form model, log-linearize around the steady-state. This linearization process is outlined in the Appendix. 


\subsubsection{Results}

Consistent with (Benhabib and Farmer 1996) (BF), we choose $\delta=.025$, $\beta=.99, m=.35$ and $n=.65$. We analyze numerically a lattice over a subregion of $(\theta, \chi)$-space roughly corresponding to the region presented in (Benhabib and Farmer 1996). ${ }^{17}$

\section{FIGURE 3 ABOUT HERE}

The results of our analysis are presented in Figure 3 and the lattice points are marked as in Figure 1. Points to the west of the region of indeterminacy correspond to determinate models.

$\mathrm{BF}$ suggest values of $\chi=0$ and $\theta=.2$. According to Figure 3 , the corresponding model would be indeterminate and only general form representations would exist. Again, we see that near the boundary of the region of indeterminacy, the CF-solutions exist. None of the points analyzed yielded a stable representation.

\subsection{The Stability Puzzle}

While there exist reduced form models that yield stable sunspots, corresponding structural models remain elusive. This apparent incompatibility is the stability puzzle. To analyze more carefully the relationship between indeterminacy and stability we consider the evolution of these characteristics while varying one parameter in the BF-model. Specifically, we hold $\chi$ fixed at zero and let $\theta$ increase from zero so that the model transitions from determinacy to indeterminacy, and we consider the stability of the REE as this transition takes place.

Recall that, when the model is determinate, the unique stationary REE is given by a particular (minimal state variable) common factor solution. Thus, eliminating $f$ from the PLM (10), we can analyze the stability of the determinate REE by analyzing the associated CF-representation.

\section{FIGURE 4 ABOUT HERE}

This observation was used to produce Figure 4. The top panel of Figure 4 represents the maximum modulus of the eigenvalues of $F^{-1} H$; the model is

\footnotetext{
${ }^{17}$ Our region of indeterminacy differs slightly from theirs because while they consider a continuous time model, ours is discrete.
} 
indeterminate if and only if this modulus is less than one, and indeterminacy obtains for $\theta$ greater than approximately .11. The model's equilibria are stable under learning provided the real part of the eigenvalues of $D T$ are less than one. The bottom panel plots the minimum among representations of the maximum of this real part. ${ }^{18}$ Notice that throughout most of the determinate region, the CF-representation is stable under learning. As $\theta$ approaches .1 a singularity is crossed beyond which the CF-representation becomes unstable. This qualitative relationship repeats itself for other parameter values and for other models; determinate solutions are typically stable, and then, as the boundary of indeterminacy is approached, the unique REE becomes unstable.

\section{Results for Alternative Information Assump- tion}

In the above analysis, we assumed that agents do not use current consumption to forecast future consumption. Alternatively, we can suppose that agents forecast $c_{t+1}$ using $c_{t}$ as well, in which case, $E_{t}^{*} c_{t+1}$ and $c_{t}$ are determined simultaneously. For the general form PLM expectations are then given by

$$
\begin{aligned}
& E_{t}^{*} c_{t+1}=a c_{t}+b k_{t}+c+d v_{t} \\
& E_{t}^{*} k_{t+1}=d_{1} k_{t}+d_{2} c_{t}-g_{2} v_{t},
\end{aligned}
$$

while for common factor PLMs $E_{t}^{*} k_{t+1}$ is as above and $E_{t}^{*} c_{t+1}$ is given by

$$
E_{t}^{*} c_{t+1}=a c_{t}+b k_{t}+c+d v_{t}+f \lambda_{i} \xi_{t} .
$$

Note that in an REE the previous information assumption and the alternative information discussed in this section are identical, since in an REE $c_{t}$ and $k_{t}$ are exact functions of predetermined variables and the observable exogenous variables. However, learning takes place outside of a rational expectations equilibrium and thus this assumption can have a non-trivial impact on stability.

To analyze stability when $c_{t}$ is in the information set, we can specify our PLMs as before; see equations (10) and (19). However, the T-map is different

\footnotetext{
${ }^{18}$ In case of indeterminacy there are possibly three representations obtained as fixed points of the T-map whereas the determinate model has only one.
} 
and is given as follows: let $\Omega=f_{1} d_{1}+f_{2} b-e_{1}$ and $\Lambda=1-f_{1} d_{2}-f_{2} a$. Then

$$
\begin{aligned}
a & \rightarrow d_{2} \Lambda^{-1} \Omega \\
b & \rightarrow d_{1} \Lambda^{-1} \Omega \\
c & \rightarrow \Lambda^{-1} f_{2} c \\
d & \rightarrow-g_{2} \Lambda^{-1} \Omega \\
e & \rightarrow \Lambda^{-1}\left(f_{2}(d+\rho e)-f_{1} g_{2}+g_{1}\right) \\
f & \rightarrow \Lambda^{-1} f_{2} \lambda f
\end{aligned}
$$

where, in the general form case, $\lambda=0$, and in the common factor case, $\lambda=\lambda_{i}$.

When evaluated at $a=a_{0}$, we have $\Lambda=0$ so that the T-map is not defined at the point corresponding to the general form representation. This does not necessarily prevent the general form solution from being stable under learning; the T-map could still direct perceptions toward this singularity. However, (32) shows that in fact this singularity is unstable.

The fixed points of the T-map are exactly the common factor representations. As usual, stability is assessed by considering the derivative of the T-map. We have

$$
D T_{a b}=\left[\begin{array}{ll}
f_{2} d_{2} \Omega \Lambda^{-2} & f_{2} d_{2} \Lambda^{-1} \\
f_{2} d_{1} \Omega \Lambda^{-2} & f_{2} d_{1} \Lambda^{-1}
\end{array}\right]
$$

and

$$
\begin{aligned}
& D T_{c}=\Lambda^{-1} f_{2} \\
& D T_{d}=0 \\
& D T_{e}=\Lambda^{-1} f_{2} \rho
\end{aligned}
$$

As with the earlier information assumption, we first investigate stability for the unrestricted reduced form using a coarse lattice. Besides the analytic result that the GF-solutions are always unstable, we find the following:

1. There are parameter regions in which neither of the $\mathrm{CF}$-solutions is stable.

2. There are parameter regions in which exactly one CF-solution is stable.

3. There are parameter regions in which both $\mathrm{CF}$ solutions are stable. 
In addition we find that there are regions in which a CF-solution is stable under both information assumptions.

For each of the structural models analyzed above, we now reconsider the stability of the common factor REE. In particular, we re-analyze each lattice of parameter values and report the relevant properties of the associated REE. The results are identical with those described earlier, except for points marked by "O" in Figures 1, 2 and 3. These represent CF solutions that are now stable under learning, though they were unstable under the previous information assumption. ${ }^{19}$

Thus corresponding to each model are several parameter values that yield stable common factor solutions. Furthermore in the SGU model the calibrations for US and Canada are tantalizingly close to stable CF-solutions. However, for each of the models the region of stable CF-solutions is a very thin subset of the indeterminacy region. Our stability puzzle can thus be restated as follows: why do the structural models have such limited areas of stability within the indeterminacy region, and why do even these stability results depend delicately on the information assumptions?

\section{Conclusions}

One approach to explaining the business cycle phenomenon is that it is at least in part due to self-fulfilling fluctuations, formally modeled as rational expectations equilibria depending on extraneous exogenous variables known as sunspots. Such solutions can be reached through natural adaptive learning rules provided an appropriate stability condition is satisfied, and examples of stable sunspot solutions have been provided in the theoretical literature.

The current paper has examined whether the required stability conditions hold for sunspot solutions in several prominent applied macroeconomic models that use non-convex versions of standard RBC models to generate indeterminacy. To examine the stability question we have shown how to apply the common factor approach to obtain new representations of sunspot solutions in a bivariate reduced form that includes the models of interest, and we have tested both these common factor sunspot solutions and the standard

\footnotetext{
${ }^{19}$ Intriguingly, for each stable CF-representation discovered, the following holds: the derivative of $D T_{a b}$ evaluated at the stable $C F$-representation has one eigenvalue equal to (or at least within $10^{-6}$ of) zero, and this zero eigenvalue appears to be robust to small changes in the parameter values.
} 
general form solutions for stability under learning. In addressing this question we have also considered two alternative information assumptions for out of equilibrium learning behavior.

Our answer to the stability question is both striking and puzzling. For the unrestricted reduced form there are large parameter regions in which sunspot solutions are stable. Furthermore, common factor sunspots are stable in many cases in which general form sunspots are not, extending our earlier findings for a univariate framework. However, when the reduced form parameters are restricted to agree with the calibrated structural models the stability results are almost wholly negative: under one of our information assumptions, none of the sunspot solutions are stable under learning, while under the alternative information assumption only small parts of the indeterminacy region have stable sunspots. Our results lead to the puzzle: why are sunspot solutions stable under learning in such small parts of the parameter space and why is the stability, even there, sensitive to the information assumptions?

Although we have cast these results in a negative light, they could be reinterpreted as a challenge to construct calibrated versions of nonconvex RBC models that are stable under learning. That this may indeed be possible is indicated by our equally striking positive stability results for the unrestricted reduced form model. Finally, we remark that the common factor technique introduced here can be generalized and applied to other multivariate frameworks. This is the subject of current research. 


\section{Appendix}

To obtain the REE given a system of nonlinear expectational difference equations it is standard to log-linearize the model about a steady-state and interpret the transformed variables as percent deviation from mean. For completeness we outline the log-linearizations of our models in this Appendix.

\subsection{General Method}

Following (Evans and Honkapohja 2001), we begin by considering the general model

$$
f\left(Y_{t}\right)+E_{t} g\left(Y_{t+1}\right)=0 .
$$

Letting $f_{i}=\partial f / \partial Y_{i}$ and $f(\bar{Y})+g(\bar{Y})=0$, the first order Taylor expansion about $\bar{Y}$ yields

$$
\text { constant }+\sum_{i=1}^{n} f_{i}(\bar{Y}) Y_{i t}+\sum_{i=1}^{n} g_{i}(\bar{Y}) E_{t} Y_{i t+1}=0 .
$$

Letting $y_{i t}=\log \left(Y_{i t} / \bar{Y}_{i}\right)$ and using $\exp \left(y_{i t}\right) \approx y_{i t}+1$ yields

$$
\sum_{i=1}^{n} \bar{Y}_{i} f_{i}(\bar{Y}) y_{i t}+\sum_{i=1}^{n} \bar{Y}_{i} g_{i}(\bar{Y}) E_{t} y_{i t+1}=0 .
$$

\subsection{FG-Linearization}

Our first application is to the structural equations of the Farmer-Guo model: see (29). The steady state is determined by the following system of equations:

$$
\begin{aligned}
\bar{K} & =1 / \delta(\bar{Y}-\bar{C}) \\
\bar{Y} & =\bar{K}^{\mu} \bar{L}^{\nu} \\
\bar{C} & =n \bar{Y} / \bar{L} \\
1 & =\beta(1-\delta+m \bar{Y} / \bar{K}) .
\end{aligned}
$$

Applying the log-linearization technique yields a linear system of expectational difference equations in four endogenous variables. This system may be reduced to obtain

$$
\begin{aligned}
c_{t} & =\left(1+\frac{\nu}{1-\nu} \beta m \bar{Y} / \bar{K}\right) E_{t} c_{t+1}+\beta m \bar{Y} / \bar{K}\left(1-\frac{\mu}{1-\nu}\right) E_{t} k_{t+1} \\
k_{t} & =-\left(\bar{C} / \bar{K}+\bar{Y} / \bar{K} \frac{\nu}{1-\nu}\right) c_{t}+\left(1-\delta+\bar{Y} / \bar{K} \frac{\nu}{1-\nu}\right) k_{t} .
\end{aligned}
$$




\subsection{SGU-Linearization}

We now apply the technique to the structural equations given by (30). Let $\theta=1+(R-1) m$. The steady state is determined by the following system of equations:

$$
\begin{aligned}
g & =\bar{\tau}(\theta \bar{Y}-R \delta \bar{K}) \\
\bar{Y} & =\bar{K}^{m} \bar{L}^{1-m} \\
\overline{C L}^{\chi+1} & =(1-m) \bar{Y}(1-\bar{\tau}) \\
\bar{Y} & =\delta \bar{K}+\bar{C}+g \\
1 & =\beta(1+(m \bar{Y} / \bar{K}-\delta)(1-R \bar{\tau})) .
\end{aligned}
$$

Using these values, the log-linearization technique yields

$$
\begin{aligned}
0 & =\tau_{t}+\frac{\theta \bar{\tau} \bar{Y}}{g} y_{t}-\frac{\delta R \bar{\tau} \bar{K}}{g} k_{t} \\
y_{t} & =c_{t}+(1+\chi) l_{t}+\frac{\bar{\tau}}{1-\bar{\tau}} \tau_{t} \\
c_{t} & =E_{t} c_{t+1}+\beta m \bar{Y} / \bar{K}(1-R \bar{\tau}) E_{t}\left(k_{t+1}-y_{t+1}\right)+\beta R \bar{\tau}(m \bar{Y} / \bar{K}-\delta) E_{t} \tau_{t+1} \\
y_{t} & =m k_{t}+(1-m) l_{t}+v_{t} \\
k_{t} & =\bar{Y} / \bar{K} y_{t}+(1-\delta) k_{t}-\bar{C} / \bar{K} c_{t} .
\end{aligned}
$$

These five equations can be combined to eliminate the variables $y, \tau$, and $l$, leading to the reduced form model.

\subsection{BF-Linearization}

Finally, we apply this technique to the structural equations given by (31). The steady state is determined by the following system of equations:

$$
\begin{aligned}
\bar{I} & =\delta \bar{K} \\
\bar{Y} & =\bar{C}^{1 /(1+\theta)}+\bar{I}^{1 /(1+\theta)} \\
\bar{Y} & =\bar{K}^{m} \bar{L}^{n} \\
n \bar{Y} & =\bar{C}^{1 /(1+\theta)} \bar{L}^{1+\chi} \\
1 & =\beta\left(1-\delta+m(\bar{Y} / \bar{K}) \bar{I}^{\theta /(1+\theta)}\right) .
\end{aligned}
$$


Using these values, the log-linearization technique yields

$$
\begin{aligned}
k_{t+1} & =(1-\delta) k_{t}+(\bar{I} / \bar{K}) i_{t} \\
y_{t} & =m k_{t}+n l_{t}+v_{t} \\
y_{t} & =(1+\theta)^{-1} \bar{C}^{1 /(1+\theta)} / \bar{Y} c_{t}+(1+\theta)^{-1} \bar{I}^{1 /(1+\theta)} / \bar{Y} i_{t} \\
y_{t} & =(1+\theta)^{-1} c_{t}+(1+\chi) l_{t} \\
\theta i_{t}+c_{t} & =\beta(1-\delta) \theta E_{t} i_{t+1}+E_{t} c_{t+1}+\beta(1+\theta) m \bar{I}^{\theta /(1+\theta)} \bar{Y} / \bar{K}\left(E_{t}\left(k_{t+1}-y_{t+1}\right)\right) \\
v_{t} & =\rho v_{t-1}+s_{t} .
\end{aligned}
$$

These six linear equations can be combined to eliminate the variables $l, y$, and $i$ leading to the reduced form model (1). 


\section{References}

Azariadis, C. (1981): "Self-Fulfilling Prophecies," Journal of Economic Theory, 25, 380-396.

Benhabib, J., And R. A. FARmer (1994): "Indeterminacy and Increasing Returns," Journal of Economic Theory, 63, 19-41.

Benhabib, J., and R. E. Farmer (1996): "Indeterminacy and SectorSpecific Externalities," Journal of Monetary Economics, 37, 421-443.

Blanchard, O., and C. Kahn (1980): "The Solution of Linear Difference Models under Rational Expectations," Econometrica, 48, 1305-1311.

Bray, M., And N. SAVin (1986): "Rational Expectations Equilibria, Learning, and Model Specification," Econometrica, 54, 1129-1160.

Cass, D., and K. Shell (1983): "Do Sunspots Matter?," Journal of Political Economy, 91, 193-227.

Evans, G. W., and S. Honkapohja (1992): "On the Robustness of Bubbles in Linear RE Models," International Economic Review, 33, 1-14.

(2001): Learning and Expectations in Macroeconomics. Princeton University Press, Princeton, New Jersey.

(2002): "Expectational Stability of Stationary Sunspot Equilibria in a Forward-looking Model," Journal of Economic Dynamics and Control, forthcoming.

Evans, G. W., S. Honkapohja, and R. Marimon (2001): "Stable Sunspot Equilibria in a Cash in Advance Economy," mimeo.

Evans, G. W., and B. McGough (2002): "Stable Sunspot Solutions in Models with Predetermined Variables," mimeo.

FArmer, R. E. (1999): The Economics of Self-Fulfilling Prophecies, Second edition. MIT Press, Cambridge, Mass.

Farmer, R. E., And J.-T. Guo (1994): "Real Business Cycles and the Animal Spirits Hypothesis," Journal of Economic Theory, 63, 42-72. 
Guesnerie, R. (1986): "Stationary Sunspot Equilibria in an N-commodity World," Journal of Economic Theory, 40, 103-128.

Marcet, A., and T. J. Sargent (1989): "Convergence of Least-Squares Learning Mechanisms in Self-Referential Linear Stochastic Models," Journal of Economic Theory, 48, 337-368.

McCallum, B. (1983): "On Nonuniqueness in Linear Rational Expectations Models: An Attempt at Perspective," The Journal of Monetary Economics, 11, 134-168.

Packalén, M. (1999): "On the Learnability of Rational Expectations Equilibria in Three Business Cycle Models," mimeo, University of Helsinki.

Rudanko, L. (2002): "On the Implications of a Balanced Budget Rule - An Adaptive Learning Perspective," Working paper, University of Helsinki.

Schmitt-Grohé, S., And M. Uribe (1997): "Balanced-Budget Rules, Distortionary Taxes, and Aggregate Instability," Journal of Political Economy, 105, 976-1000.

Shell, K. (1977): "Monnaie et Allocation Intertemporelle," Working paper, CNRS Seminaire de E.Malinvaud, Paris.

Woodford, M. (1990): "Learning to Believe in Sunspots," Econometrica, $58,277-307$. 


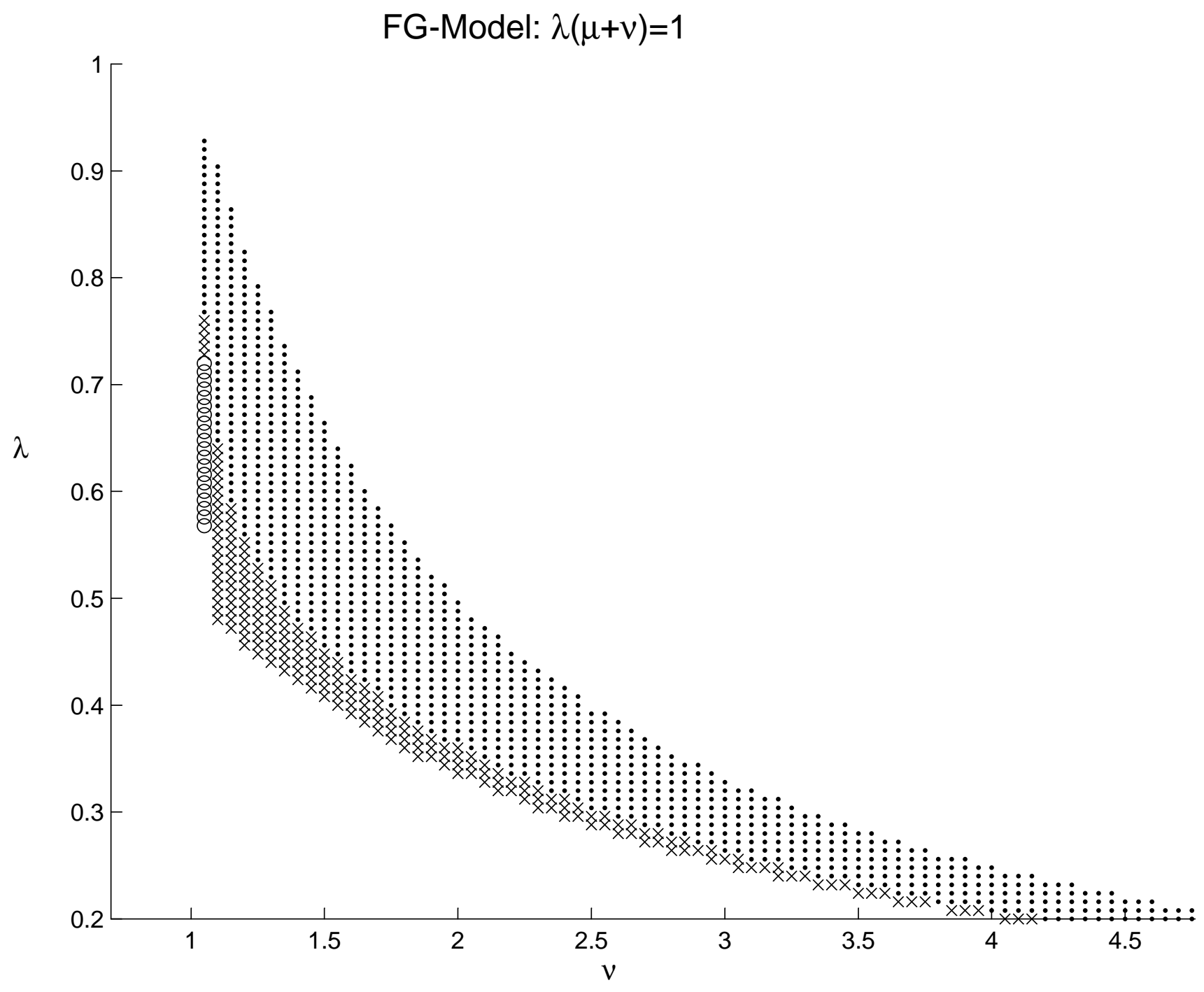

Figure 1 


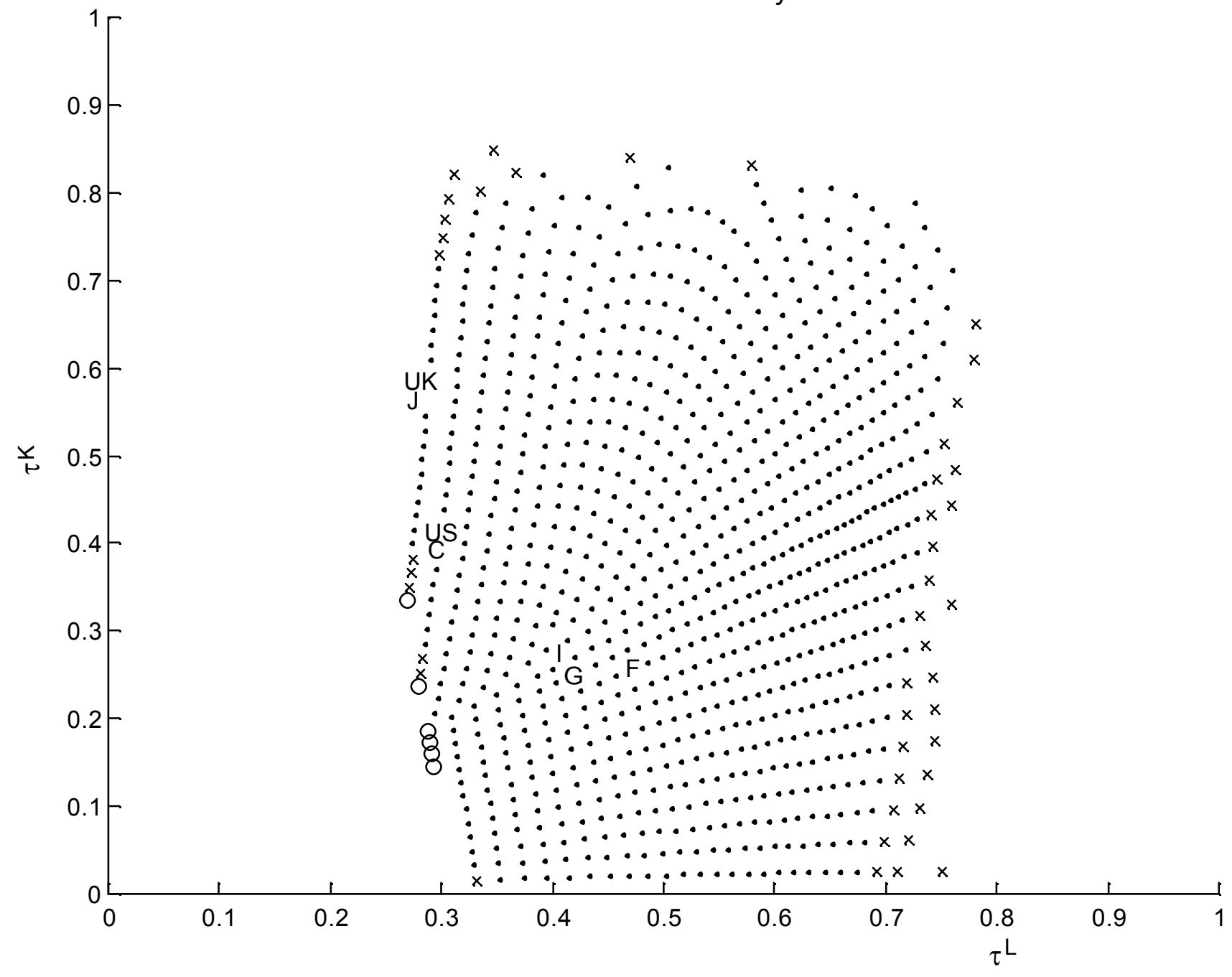

Figure 2 


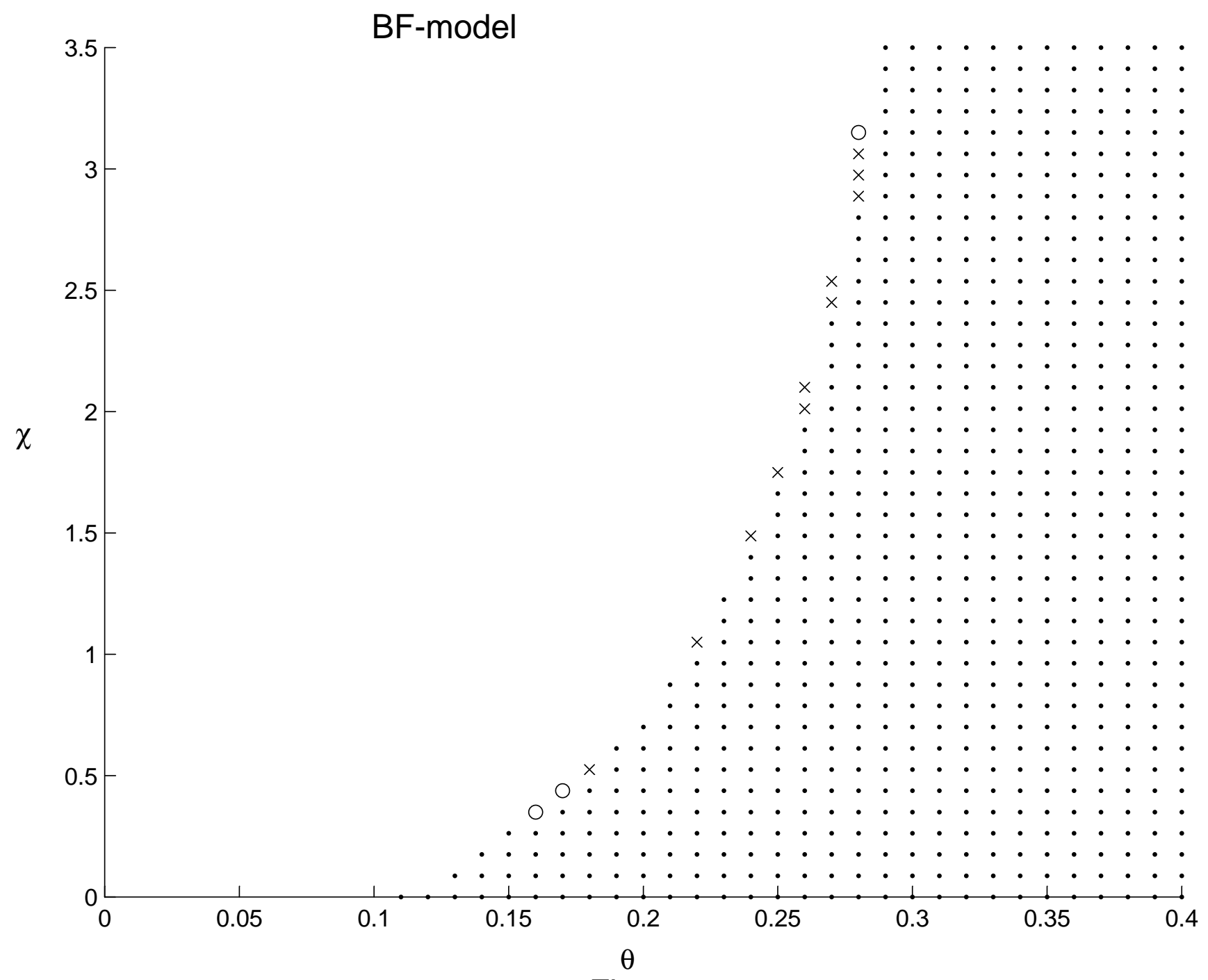

Figure 3 

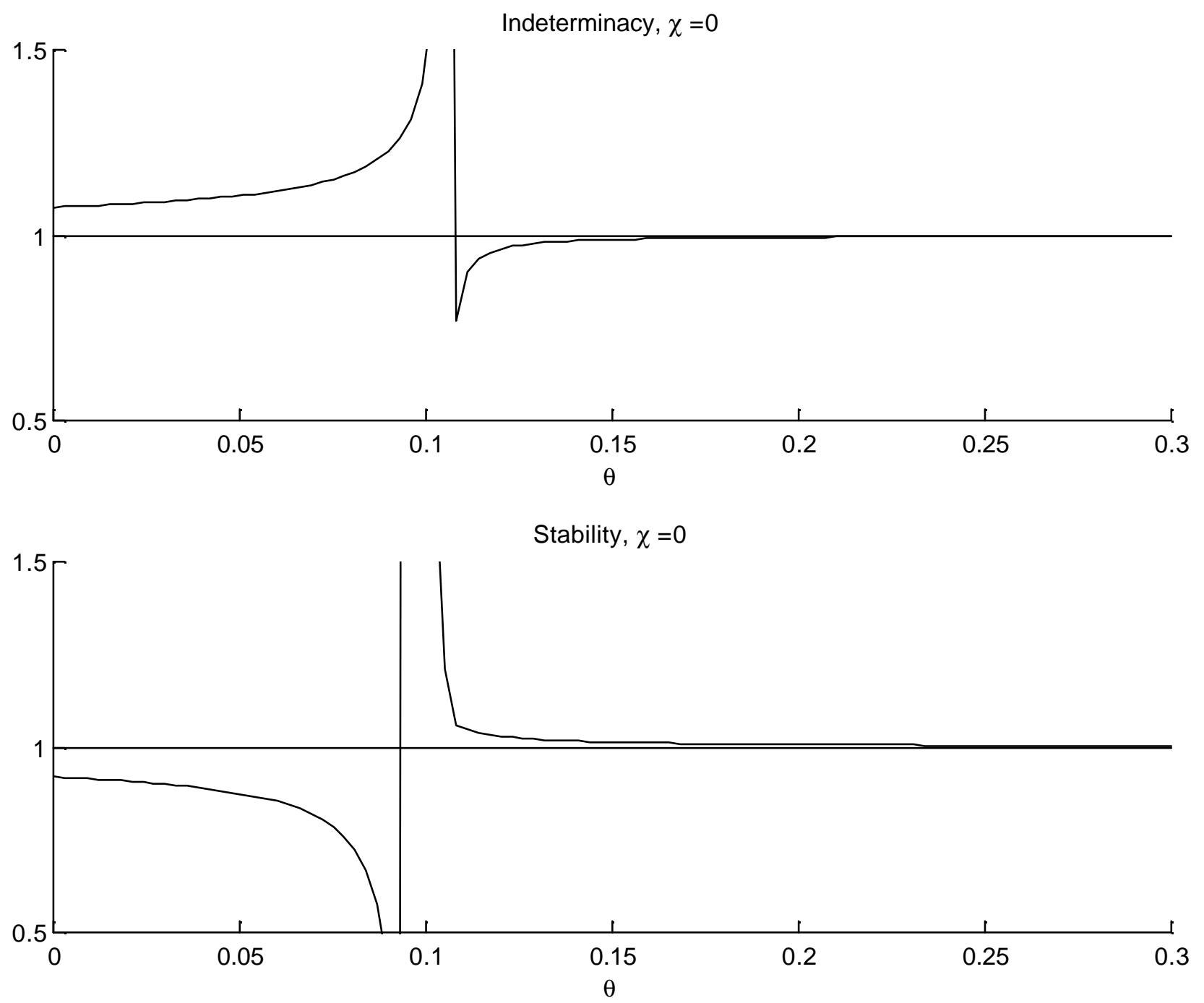

Figure 4 